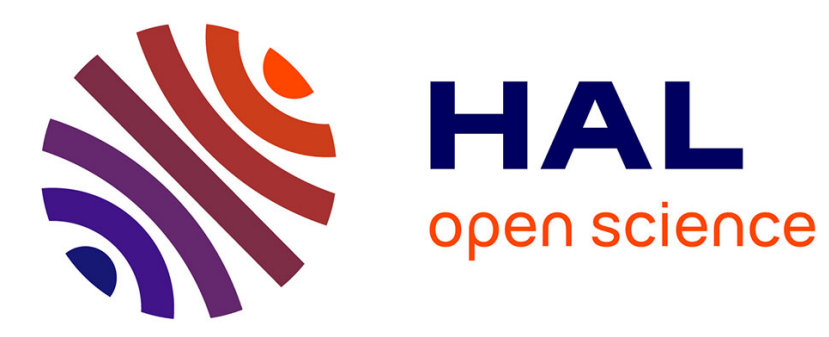

\title{
Intelligent products through a SOHOMA prism
}

\author{
William Derigent, Duncan Mcfarlane, Hind Bril El-Haouzi
}

\section{To cite this version:}

William Derigent, Duncan Mcfarlane, Hind Bril El-Haouzi. Intelligent products through a SOHOMA prism. 10th Workshop on Service Oriented, Holonic and Multi-Agent Manufacturing Systems for Industry of the Future, SOHOMA 2020, Oct 2020, Paris, France. hal-03018048

\section{HAL Id: hal-03018048 \\ https://hal.science/hal-03018048}

Submitted on 21 Nov 2020

HAL is a multi-disciplinary open access archive for the deposit and dissemination of scientific research documents, whether they are published or not. The documents may come from teaching and research institutions in France or abroad, or from public or private research centers.
L'archive ouverte pluridisciplinaire HAL, est destinée au dépôt et à la diffusion de documents scientifiques de niveau recherche, publiés ou non, émanant des établissements d'enseignement et de recherche français ou étrangers, des laboratoires publics ou privés. 


\title{
Intelligent Products through a SOHOMA prism
}

\author{
William DERIGENT ${ }^{1}$, Duncan MCFARLANE ${ }^{2}$, Hind BRIL EL HAOUZI ${ }^{1}$ \\ ${ }^{1}$ CRAN CNRS UMR 7039, Université de Lorraine \\ Campus Sciences, BP70239, \\ 54506 Vandœuvre-lès-Nancy, France \\ \{firstname. lastname\} @univ-lorraine.fr \\ ${ }^{2}$ Institute for Manufacturing \\ Cambridge University Engineering Department, \\ 17 Charles Babbage Road, \\ Cambridge, CB3 OFS, UK \\ dcmeng.cam.ac.uk
}

\begin{abstract}
In the framework of the SOHOMA 2020 special session "SOHOMA $10^{\text {th }}$-year anniversary", this paper aims to make a review of the evolution of one important concept studied in the SOHOMA community, namely the Intelligent Product concept. This paper is not review of Intelligent Products - there are several of these already - but rather examines the history of the development of this concept through the $1^{\text {st }}$ to $9^{\text {th }}$ editions of SOHOMA, while also proposing future developments on this concept.
\end{abstract}

Keywords: Intelligent Products, History

\section{Introduction}

In the framework of the IMS (Intelligent Manufacturing Systems) community, and as demonstrated by the Auto ID Centre developments in 2000-2003, the related concepts of Internet of Things and Intelligent Products sought to connect physical objects with digital information and even "intelligence" associated with the object. Indeed, substantial information distribution improves data accessibility and availability compared to centralized architectures. Product information may be allocated both within fixed databases and/or within the product itself, thus leading to products with informational and/or decisional abilities, referred as "Intelligent Products". This concept has been widely discussed over more than two decades, beginning in 1997when several authors separately presented the notion of product intelligence to describe an alternative, product-oriented way in which supply chains and automated manufacturing might work (see

adfa, p. 1, 2011.

(C) Springer-Verlag Berlin Heidelberg 2011 
[1]-[4]. The models proposed described manufacturing and supply chain operations in which parts, products or orders (collections of products) would monitor and potentially influence their own progress through the industrial supply chain. The supply chain model based around product intelligence provided a conceptual focus for these developments. A very simple search on Google Scholar ${ }^{1}$ of articles related to this concept reveals that more than 300 papers have been published on the subject since 2000 . This number does not include articles related to "intelligent product" design (more related to the mechanical engineering field or the smart "PSS" field). Many different definitions of "Intelligent Products" have been proposed. A comparison of the different types is provided in [5]. Reviews also exist [6]. The objective of this paper is not to produce yet another review on Intelligent Products. In the framework of SOHOMA 2020, it rather aims at underlining 1) how the SOHOMA community helped to develop and spread this concept, via scientific proposals and industrial applications, 2) how the SOHOMA community is shaping the future of the intelligent product concept.

\section{Methodology applied in this paper}

SOHOMA contributions related to the domain of the "Intelligent Product" have been analyzed from SOHOMA 11 to SOHOMA 19 via a careful exploration of the proceedings. In our point of view, the concept of Intelligent Product is mainly related to 2 subfields : The first one is the asset management thanks to the Intelligent Products which has been extensively studied, leading to advanced asset management systems, most of the time dedicated to product tracking in logistics. A second important use of the intelligent product concept is the product-driven control of manufacturing systems, where the product is an actor of its future in the manufacturing process. Both aspects are, from our point of view, two important and complementary aspects of the same concept. Both are moreover needed requirements to ensure the resilience of production systems [7].

The first step of the methodology is thus to extract relevant papers from the proceedings. Each paper related to one of the previous concepts (or in a session related to the Intelligent Product) was taken into account, and keywords saved. Citation numbers were extracted from Google Scholar. As our objective is to process this extraction via adapted tools, keywords have been 'normalized'. Indeed, there are many keywords that describe identical to very similar concepts (e.g. product-driven control, product-driven automation and product-driven systems). The raw extraction is cleansed and completed before the next step.

The second step aims to define a conceptual map called a co-occurrence network. Co-occurrence networks are generally used to provide a graphic visualization of potential relationships between people, organizations, concepts, biological organisms or other entities represented within written material. The generation and visualization of co-occurrence networks has become practical with the advent of electronically stored text compliant to text mining ${ }^{2}$. In this paper, a co-occurrence network based on similar keywords in the extracted papers is produced and analyzed.

\footnotetext{
${ }^{1}$ www.scholar.google.fr / search: allintitle: "intelligent products" OR "intelligent product"

${ }^{2}$ https://en.wikipedia.org/wiki/Co-occurrence_network
} 
The next section first introduces the general co-occurrence network and details the different identified clusters in different subsections. The last part of this section uses the form of this network to foresee a possible future trend in the Intelligent Product field.

\section{History of the intelligent product concept in SOHOMA}

Intelligent products (IP) are present in SOHOMA from the beginning as showed in table1 (presented in the annexes). This table lists per year all the papers extracted from SOHOMA Proceedings, with a total of 49 papers. However, the number of publications is not even through time, as depicted fig. 1, going from 2 (SOHOMA 2017) to 8 (SOHOMA 2014). The proportion of these papers above the total number of SOHOMA papers goes from $24 \%$ in 2011 to $28 \%$ in 2014 and then goes down to $11 \%$ in 2019 . Each paper in this field has been cited $\sim 6$ times, going up to 29 times for [8]. In the different proceedings, three parts are specifically dedicated to the IP (SOHOMA 2012 -Part II 'Intelligent Products'; SOHOMA 2013 - Part IV 'Intelligent Products'; SOHOMA 2015 - Part I 'Application of Intelligent Products') and two parts contains works related to the IP (SOHOMA 2014 - Part IV 'Physical Internet Containers; SOHOMA 2015 - Part II 'Recent Advances in Control for Physical Internet and Interconnected Logistics').

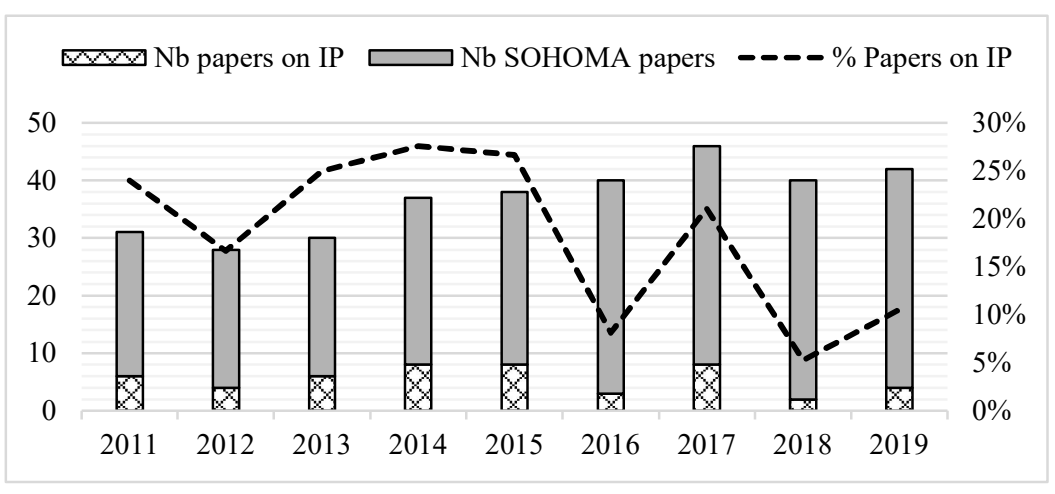

Fig. 1. Overview of the number of SOHOMA Papers on Intelligent Products

The co-occurrence network based on keywords is done via an automatic processing executed via VOSviewer, a specific software tool dedicated to bibliometrics network. Other tools exist like $R$ and its bibliometrics package, Pajek, Sci2, Cytoscape, etc. VOSviewer has been selected due to its user-friendly interface and its ability to process easily bibliographic databases ${ }^{3}$. This network is presented on fig. 3 where it is clear that these clusters are strongly interrelated yet still have independent threads. It is composed

\footnotetext{
${ }^{3}$ Note that the complete list of references in *.bibtex/*.ris format as well as the VOSviewer files have been provided as supplementary material with the article submission.
} 
of nodes representing the different paper keywords, and links representing relations between these nodes. The shorter the links, the more the keywords are used in the same papers. It is then possible to define clusters of keywords, based on their co-occurrence distance. In this paper, these clusters are interpreted as domains or categories in the IP research field. The network thus shows a total of 6 clusters:

- Cluster 1: Product-Driven Systems (PDS) ${ }^{4}$

- Cluster 2: Product Lifecycle Information Management (PLIM)

- Cluster 3: Physical Internet (PI)

- Cluster 4: Multi-Agent Systems (MAS)

- Cluster 5: Internet of Things (IoT)

- Cluster 6: Digital Twin (DT)

One first analysis would be on the type of the different clusters. Indeed, clusters 1 to 3 are related to research works originated from works in the IP field while clusters 4 to 5 are more related to theory, methods and tools used to realize the IP concept.

Cluster 6 is far from the others, meaning it is not too interlinked with the other ones. It can thus be interpreted as a relatively new cluster and new research field as well. The annual distribution of IP papers in each cluster is shown fig. 2. It can be seen that, from the beginning, papers have been published on PDS and PLIM themes in the SOHOMA conferences. In this respect, the theme PI within the framework of IP has been addressed later, in 2014. The more recent cluster is the DT one, where papers have been proposed from 2018 .

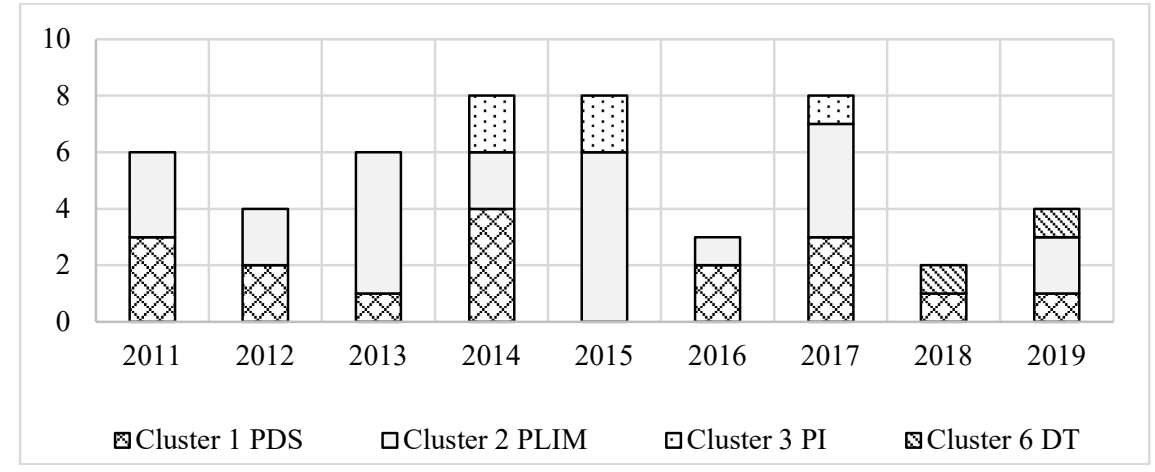

Fig. 2. Annual distribution of IP papers in each cluster

\footnotetext{
${ }^{4}$ PDS and ODS (Order-Driven Systems) are often used interchangeably
} 


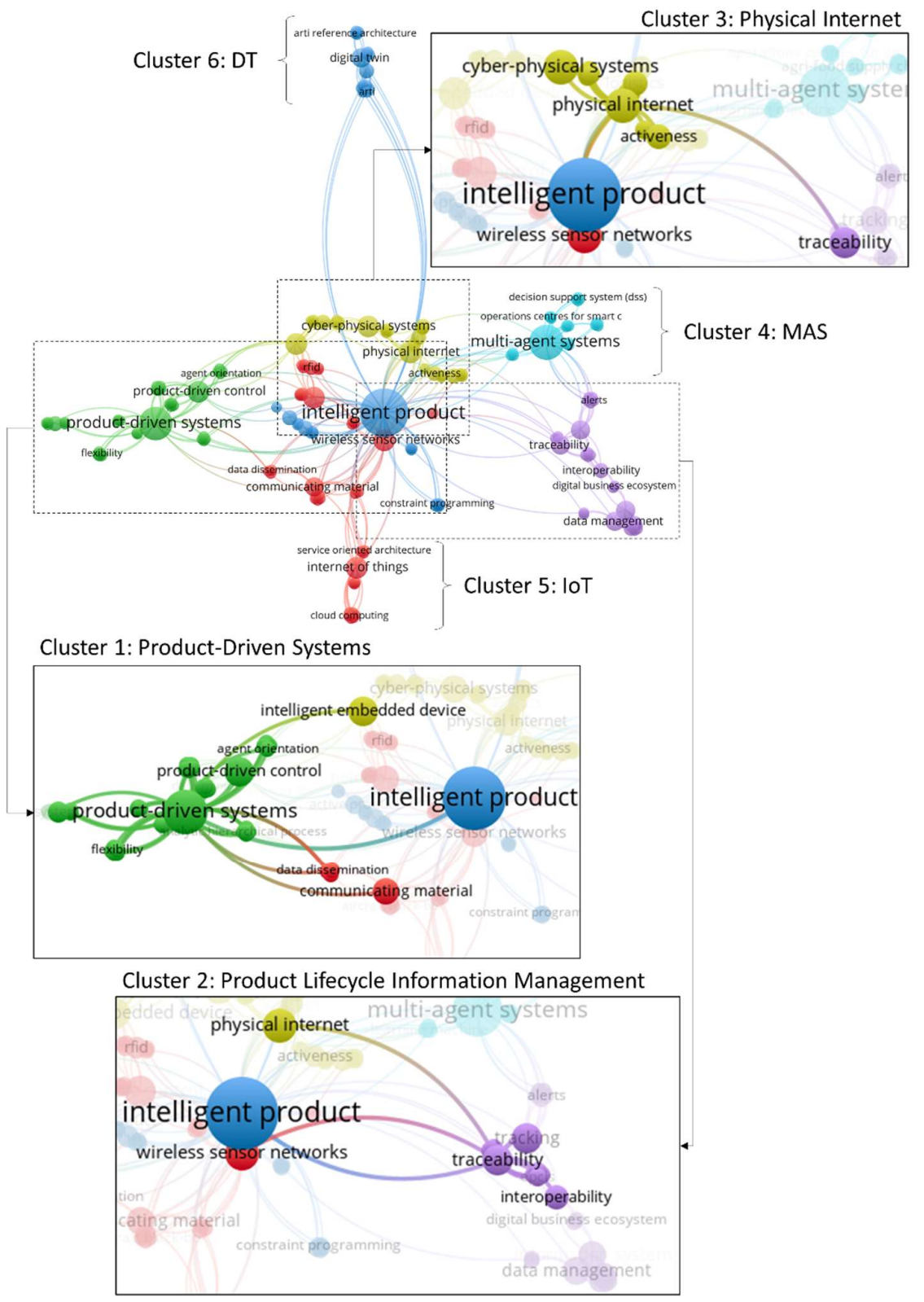

Fig. 3. Intelligent Product related keywords co-occurrence network obtained from SOHOMA proceedings 
In the rest of this section, the different clusters are detailed, focusing on the timeline of the related research works. Only the clusters related to core SOHOMA research fields are tackled. Each reference cited hereafter is coming from SOHOMA conference proceedings.

\subsection{Cluster 1: Product-Driven Systems}

Product-Driven Systems are defined in [9] as a way to optimize the whole product lifecycle by dealing with products, whose informational content is permanently bound to their virtual or material contents and thus are able to influence decisions made about them, participating actively to different control processes in which they are involved throughout their life cycle. They introduce some examples highlighting the way PDS can improve the global product lifecycle performance in the design phase, production phase or use phase. Designing a PDS is a challenge involving three fundamentals aspects: functions, architecture, and interactions.

Intelligent products are the core of a PDS. The functional features given to the intelligent products in the PDS are then essential when designing the PDS. Research works in this area are also intertwined with Cluster 2 and will therefore be described later. The architecture of a PDS in another facet of the PDS design and many research works address this issue. In particular, [10] present the PROSIS framework, an evolution of PROSA, where the Staff Holon is replaced by a Simulation. In this Isoarchic architecture, Ambient Control Entity (ACE), located near the resource Holon, allows each I Holon (informational part of a Product Holon) to call ambient services related to decision making or communication. Guided by the idea that all distributed intelligent approaches are mimicking nature and human behavior, several researchers also explored bio-inspiration to build PDS. For example, [11] define a control architecture based on the Viable System Model called PDS-VSM exhibiting interesting recursive properties as natural structures like living organisms do.

Bio-inspiration has been also widely used in the framework of the third and last aspect of a PDS, i.e interactions. Several bio-inspired approaches have been proposed in the past, as Ant Colony Optimization and the Firefly Algorithm. [12] propose a mechanism inspired from the stigmergy using the notion of volatile knowledge. Mobile products can share with each other information (knowledge) about their environment, whose confidence decrease through time. This mechanism allows to propagate knowledge about perturbations and to return to normal situation in a distributed way without a coordinator. Negotiation approaches are investigated in [13]. They propose a negotiation heuristic based on the notion of critical ratio ((Due_date - current_date) / total shop time remaining). Products are negotiating their schedules with other products by exchanging this value. The collaboration mechanism between Intelligent Products can also be formalized using Multi-criteria Decision Making (MCDM) techniques. In [10], [14], the collaboration mechanism is based on the AHP/ANP (Analytic Hierarchy Process / Analytic Network Process).

PDS have pros and cons. They are highly agile and reactive, and they allow for a potentially greater involvement of the end customer. However, they are not widely accepted in industries because of the lack of performance proofs, mainly due to their 
myopic behavior, allowing them to make decisions locally efficient but inconsistent with the global objective. This nevertheless depends highly on the use case and working conditions. Indeed, performances of a distributed architecture can be as good as with a centralized driving solution as stated in [15]. To help PDS to be less myopic, [16] illustrates how the centralization of data via a discrete-event observer can help to achieve better decisions. This notions of "discrete-event observer" is an online simulation model running in parallel of the manufacturing system observed. One can note that this notion is not so far from the notion of Digital Twin that arises years later.

Another strategy to counter this myopia is to mix predictive scheduling and reactive control. A state of the art was produced in this respect [17] and several works of the SOHOMA community address this issue. [18] propose a PDS employing a scheduling rule-based evolutionary simulation-optimization strategy to dynamically select the most appropriate local decision policies to be used by the products, when disturbances appear. The originality is to choose decision policies (i.e dispatch rules) instead of fixed schedules, allowing more flexibility on the product level. Another problem is to know when to switch from the predictive scheduling to the reactive one, and when to come back. Since the decision is not binary, a fuzzy model of the switching mechanism is used in [19], [20]. A last method proposed by the SOHOMA community in the framework of PDS is to bring some robustness into the scheduling strategies, thanks to operational research works dealing with scheduling under uncertainties [21].

Systems evolve and PDS also do, meaning their architecture, configuration, states can change through time. To react as correctly as possible, a fine understanding of the system evolution is needed. Giving these learning abilities to a PDS is a central challenge in a world where manufacturing systems will constantly change and be reconfigured thanks to Industry 4.0 technologies. It is also the opportunity to transform PDS into Predictive Manufacturing Systems as defined in [22]. [23] state that the synchronization of physical and information flows in a PDS implies that large data volumes may be exploited to create the necessary knowledge and information for product decision-making. The study illustrates how product information can be processed via neural networks to obtain shop-floor knowledge, i.e. a function computing the lead time of a product from the beginning of its manufacturing to the input queue of a bottleneck. This function can then be integrated into the product memory for further uses. [24] consider that Intelligent Products should also be able to reuse their past experiences to enhance their decisional performances. Past experiences are then stored and Reinforcement Learning (here a Q-learning algorithm) is then used to select the best action a product may do in each situation.

Some industries are willing to use these techniques and some SOHOMA articles describe applications of PDS via some case studies in the manufacturing industry [25], [26].

As a conclusion, PDS are advanced manufacturing systems that can lead to a better agility and reactivity, with a better integration of the end customer. SOHOMA explored this area and bring many contributions form the proof of concept to valuable implementations in industry and generic framework architectures (PDS architectures, interactions between products, coupling of centralized and decentralized decision-making, machine learning and so on). 


\subsection{Cluster 2: Product Lifecyle Information Management}

The other facet of the Intelligent Product is related to the data. It is a cluster as important as the first one, with many contributions made in this field by the SOHOMA community. A first important research work is the paper of [27] which retraces the history of the Intelligent Products in the Supply Chain from 2002 to 2012. This paper cites the original definition of the Intelligent Product introduced in [1], [28] :

An Intelligent Product is a product (or part or order) that has part or all of the following five characteristics:

1. Possesses a unique identity

2. Is capable of communicating effectively with its environment

3. Can retain or store data about itself

4. Deploys a language to display its features, production requirements etc.

5. Is capable of participating in or making decisions relevant to its own destiny

Two levels of product intelligence are commonly defined: IP level 1 regroups features 1 to 3, while IP level 2 covers the 5 features.

The management of product information all along its lifecycle is then referred as Product Lifecycle Information Management (PLIM) and ensured by Product Lifecycle Information Management Systems as detailed in [29]. In the 2000s, the classic implementation of the intelligent product reflected the developments being made in the Cambridge AutoID centre. Indeed, a unique ID is stored on a low-cost RFID tag attached to the product. This ID can be resolved to a network pointer to a linked database and a decision-making software agent. This information is then available all along the supply chain or even all along the product lifecycle. Such systems can provide new services in different phases of the lifecycle, obviously in the manufacturing phase or logistic phase, but is not limited to. For example, as described in [30], it could be used to provide new repair services for domestic appliances, by providing appliance lifecycle data and part designs respectively to diagnostic services and 3D printing services.

PLIM systems are basically distributed data management architectures. In this regard, several works and members of the SOHOMA community highly contributed to this field. Different architectures, messaging protocols and formats have been proposed as described in [31]. The EPCIS architecture is one of the well-known distributed data management architecture, standardized by GS1 and specially adapted to product tracking in the supply chain [32]. These manufacturing concepts have been also applied in other domains, as described in [33], where the EPCIS standard has been applied to workforce management in hospitals, leading to the 'aTurnos' cloud-based solution ${ }^{6}$. DIALOG is another proposed architecture proposed by SOHOMA members, based on a multi-agent system distributed in every actor of a given supply chain. In this architecture, a specific messaging protocol initially called PMI (Product Messaging Interface) and further named QLM (Quantum Lifecycle Management) is used. As EPCIS, QLM is now a standard and is detailed in [34]. This paper also underlines why such messaging

\footnotetext{
${ }^{5}$ These two citations are not extracted from SOHOMA proceedings.

${ }^{6} \mathrm{https}: / /$ www.aturnos.com/
} 
standard are needed in Business-to-Business infrastructures and demonstrates via usecases how flexible QLM is, compared to the other existing messaging protocols.

The intelligence and as a result the information of the product can also be on the product itself. According to [35], product intelligence is not a primary function of a product, but comes as secondary functions (i.e. communicating, triggering, memorizing) that can be embedded in the product (also referred as "target system") but also available online. Because the activeness is linked with the target system, the secondary functions follow the product all along its lifecycle from manufacturing to recycling. These functions can be added or removed, moved into the target system or online, depending on the phase requirements [36]. As a result, several applications of the activeness concern different phases of the lifecycle. For example, the work of [37] applies the activeness concept during the use phase, in order to give to the products augmented monitoring and analysing functions. It has also been used in the logistic phase and applied to smart containers, as will be described later.

To store the intelligence directly on the product, many different devices can be used, not only RFID, but also micro-computers or wireless sensor nodes. Because these devices have more computing power and memory than classic RFID tags, they can execute all or part of the product secondary functions. In [38], the evolution from communicating products (products equipped with RFID tags) to autonomous products is described. The authors provide a case study of a flexible manufacturing system where products are evolving from IP level 1 to IP level 2. Indeed, transport carriers, originally equipped with RFID tags, receive a miniaturized electronic device composed of a CPU, a RFID reader, some direction actuators, sensor, and a HMI. Works in the communicating materials or Physical Internet detailed after also use Wireless Sensor Nodes (WSNs) to realize the intelligent product concept.

Communicating materials are materials equipped with micro-electronic components, either RFID tags (Kubler, Derigent, Thomas, \& Rondeau, 2011) or self-powered WSNs embedded into the material [40]. The interests of such material are diverse: (a) because of their data storing capacity, they can convey all information related to design, manufacturing and logistics, useful during the BOL (Beginning Of Life - design, manufacturing and construction) and the EOL (End Of Life - dismantlement and recycling) of a product; (b) given their ability to sense their environment and process related information, they can also be used during the MOL (Middle Of Life - exploitation and maintenance) as intelligent sensors, mainly to perform health monitoring. The material could be either wood, textile or concrete. First works dealing with communicating materials are addressing the data dissemination / data replication issues in this type of materials. Lately, the work of [41] aims to explore the monitoring capability of communicating materials, by developing concrete beams equipped with energy-aware WSNs. Indeed, this concrete beam can monitor its status in nearly real-time.

This last work shows that IP (Communicating materials and so on) can exhibit monitoring functions, and can be aggregated to build a global architecture, monitoring the whole system performances. This is another aspect of the Intelligent Product that has attracted attention in the SOHOMA community. In [42], a multi-agent system is embedded into wireless nodes, to manage a wireless data acquisition platform applied on industrial systems. In this architecture, each sink node manages a cluster of wireless sensors. Each sink node contains three interacting agents respectively responsible for configuring/reconfiguring the cluster, aggregating/filtering data and communicating 
with the previous agents/other sink nodes. This wireless architecture is illustrated on an oil and gas refinery. [43] deal with the development of data management systems for fleet of trains, able to gather, memorize, manipulate and communicate data coming from equipments. They demonstrate the interest of holonic semi-heterarchical architectures where each Holon is a product composing the whole system. From this conclusion, they develop the Surfer Data Management Architecture and apply it to train transportation [44]. In the same vein, distributed monitoring is explored in [45]. The target system is a manufacturing shop floor, where each component of a system (resource of product) is linked to a monitoring agent. These agents then send monitoring data via a monitoring data stream, which are read, aggregated, and stored by a monitoring controller agent. Monitoring controller agent can also send their data via the monitoring data stream, and its data may be aggregated by another monitoring controller agent. This way to proceed is equivalent to previous architectures and highly scalable.

In SOHOMA community, these works on PLIM have been applied in manufacturing, supply chain [46], but also in the agriculture and agri-food domain [47], in the pharmaceutical [48] and building sectors [49], [50]. Finally, Information systems dealing with intelligent products have been proposed in SOHOMA with a specific importance given to the distribution of information on the product/on the network. EPCIS, DIALOG, the activeness concept or the communicating materials are among the main contributions presented in SOHOMA conferences.

\subsection{Cluster 3: Physical Internet}

The Physical Internet has been proposed in [51] and formally defined as an open global logistics system leveraging interconnected supply networks through a standard set of modular containers, collaborative protocols and interfaces for increased efficiency and sustainability. The Physical Internet is present from the beginning of SOHOMA. First works on this theme can be found in 2011 [52], where the new paradigm is introduced. and propose a bio-inspired method to build the Open Hub Network is proposed.

A first interesting finding is that concepts of Physical Internet and Intelligent Product are not merged at the beginning, at least in works presented in SOHOMA. The first time both concepts are found merged in SOHOMA works is in [8], with the main idea to realize the notion of PI-Container (smart container used in the Physical Internet Paradigm) via the application of the activeness concept to a normal container. The article describes the concept of PI-Container activeness and detail the different needed information and interaction possibilities a "active" PI-Container should include. A later paper [53] goes deeper into this concept and propose a guide to represent and analyze collectives of PI-Containers and their inner interactions. Indeed, a simple PI-Container can be assembled with other PI-Containers to form a Composite one. Going further into the study of PI-container, [54] address the problem of composition/decomposition of PI-Containers. In their approach, they consider the PI-Container as a normal container equipped with a wireless sensor node. A Collective of PI-Containers is then equivalent to a wireless sensor network and can identify its real composition from information collected on the network. This information combined with an optimization process 
leads to a 3D map of the containers composing the collective, called a VoC (Virtualization of Container).

A second interesting finding is that concepts from the PDS cluster have also been applied to the Physical Internet. For example, the PROSIS architecture detailed previously is first applied in an intralogistics context in [55]. In this work, authors introduce the concept of Wireless Holons Network, constituted by mobile Holons (shuttles, moving products) and fixed Holons (workstations). Mobile Holon are the one equipped with a WSN mote. As in the classic PROSIS approach, ACEs (Ambient Control Entities) placed on the workstations can provide ambient services to the mobile Holons. Following this first proposal, [55], [56] address more directly the Physical Internet and propose an adaptation of the PROSIS framework to this domain.

As a conclusion, SOHOMA contributions to the Physical Internet relate to the introduction of the Intelligent Product to the Physical Internet: proposals described in the other clusters as the activeness concept or PDS architectures like PROSIS have been applied promisingly to this new field. The Intelligent Product introduction is helping to give the Physical Internet an orientation towards supporting customization as well as greater efficiencies in the supply chain.

\subsection{Cluster 6: Towards Digital Twins at the core - One of the future trends?}

Among all the clusters of interest, cluster 6 is the smallest in terms of number of papers. This cluster regroups keywords related to the virtual representation of the product and especially the keyword "Digital Twin", that has emerged recently in the manufacturing community (thus explaining the size of the cluster). Indeed, The Digital Twin is a new paradigm in simulation and modelling, defined by $[57]^{7}$ as "an integrated multi-physics, multi-scale, probabilistic simulation of a complex product [using] the best available physical models, sensor updates, etc., to mirror the life of its corresponding twin".

Moreover, as can be seen fig. 3, this cluster is the farthest from the others. It means that it has the least connection with the other clusters for the moment, in the IP community. It can be interpreted as a new field of research, interesting for IP community. However, Digital Twin has a wider spectrum than the IP community, and many other SOHOMA works and sessions have already addressed this field.

In [58], the author discusses the history of PROSA and presents its evolution named ARTI - Activity $\underline{\text { Resource }}$ Type Instance. ARTI makes a strong separation between the Intelligent Agents and Intelligent Beings, emphasizing the fact that the Intelligent Beings are describing what is more than what is decided. In the framework of Industry 4.0, the reality of these intelligent beings could be represented by Digital Twins, which then become the unique "contact persons" to access the world-of-interest. The notion of Digital Twin thus becomes crucial generally for HMS (Holonic Manufacturing System) but also for PDS. One of the first exploration of the connection between ARTI and Digital Twin reported in SOHOMA is the work of [59] in the context of semi-continuous production processes

\footnotetext{
${ }^{7}$ This citation is not issued from SOHOMA proceedings.
} 
In authors propose a dynamic adaptation process of M-BOMs (manufacturing bill of materials) based on BIM Building Information Modeling (BIM) and CPS assets in building sector. The use of BIM assets and real time follow-up based on CPS paradigm could be a source of valuable data to support the planning and monitoring activities throughout building life cycle and pave the way of the introduction the digital twin as core system for those activities.

Digital Twin also begins to play a role in smart asset management, as underlined by [60]. The authors propose a framework for future development of smart asset management during the operations and maintenance phase., integrating the concept of Digital Twin. They argue that the future framework should be divided into three layers (smart asset layer, smart asset integration layer, smart DT-enabled asset management layer). Compared to "old" PLIM systems, it appears a need to store and manage the lifecycle not only of data and information, but also of simulation models like the DTs, that should be easily connected to real-life data.

This research theme is still new in SOHOMA but merging DT and IP is already stressed by SOHOMA papers as a future interesting field. SOHOMA contributed to this merge by proposing adapted control and information management architectures.

\section{Conclusions}

During the past 20 years, a lot of different works have been done in the world of Intelligent Products and in particular, over the last 10 years systems based around the Intelligent Product concept have undoubtedly found a home in the SOHOMA series of workshops. This paper has retraced the evolution of connected topics that have been gravitating around the IP concept for decades through the lens of SOHOMA, and this accurately reflects the fact that Intelligent Products are a broad concept rather than a specific industrial solution. This study also lists the number of IP related papers produced in the SOHOMA conferences (around 50). It demonstrated that, for ten years, this notion has been a rich concept for SOHOMA, as well in production control than in data management. The SOHOMA community has participated to this scientific adventure, evidenced by the important ratio of papers dedicated to this theme, even if less important these last years.

SOHOMA members have contributed to Intelligent Product research in a number of different areas (referred to as clusters in this paper). Three main clusters have been identified, as more representative contributions, thanks to a bibliography analysis: Product-Driven Systems, Product Lifecycle Information Systems and Physical Internet For each cluster, a synthesis of the works done by the community has been provided. One last and smallest cluster is perhaps related to one of the future trends of IP - namely the rapid take up in recent times of digital twins. This concept can be seen as encompassing as that it extends the perimeter of the intelligent product to intelligent "everything" and push for the emergence of new methods (for eamplex data sciences) and new requirements of real time, observation, data ming...In addition to these clusters we have observed a number of common methods and tools being used in the 
development of intelligent product based approaches: multi agent systems, traceability approaches and the development and deployment of embedded devices.

In the past, the advent of technologies as RFID tags and WSN helped to concretize the IP concept. In the future, the envisaged development of tools and methods associated with Industry 4.0, the development of infrastructure for IoT, human-object integration in industrial operations and the development of increasingly autonomous capabilities in industrial systems will certainly provide a fantastic playground for this concept, which will still evolve thanks to the never-ending work of passioned researchers.

\section{$5 \quad$ References}

[1] C. Y. Wong, D. Mcfarlane, A. A. Zaharudin, et V. Agarwal, « The Intelligent Product Driven Supply Chain », in Proc. IEEE Int. Conf. on Systems, Man and Cybernetics, 2002, p. 4-6.

[2] D. McFarlane et Y. Sheffi, « The impact of automatic identification on supply chain operations ", Int. J. Logist. Manag., vol. 14, no 1, p. 1-17, 2003.

[3] M. Kärkkäinen, T. Ala-Risku, et K. Främling, « The product centric approach: a solution to supply network information management problems? », Comput. Ind., vol. 52, $\mathrm{n}^{\mathrm{o}}$ 2, p. 147-159, 2003.

[4] G. Morel et B. Grabot, "Special issue on intelligent manufacturing », Eng. Appl. Artif. Intell., vol. 16, nº 4, p. 271-393, 2003.

[5] D. McFarlane, V. Giannikas, A. C. Y. Wong, et M. Harrison, « Product intelligence in industrial control: Theory and practice », Annu. Rev. Control, vol. $37, \mathrm{n}^{\circ} 1$, p. 69-88, 2013.

[6] G. G. Meyer, K. Främling, et J. Holmström, « Intelligent Products: A survey », Comput. Ind., vol. 60, n 3, p. 137-148, 2009.

[7] R. Srinivasan, D. McFarlane, et A. Thorne, « Identifying the requirements for resilient production control systems », in Studies in Computational Intelligence, vol. 640, Springer, 2016, p. 125-134.

[8] Y. Sallez, B. Montreuil, et E. Ballot, « On the activeness of physical internet containers », Stud. Comput. Intell., vol. 594, p. 259-269, 2015.

[9] D. Trentesaux et A. Thomas, «Product-driven control: a state of the art and future trends », IFAC Proc. Vol., vol. 45, nº 6, p. 716-721, 2012.

[10] Y. Dubromelle, F. Ounnar, et P. Pujo, « Service oriented architecture for holonic isoarchic and multicriteria control», in Studies in Computational Intelligence, 2012, vol. 402, p. 155-168.

[11] C. Herrera, S. B. Berraf, et A. Thomas, « Viable system model approach for holonic product driven manufacturing systems », in Studies in Computational Intelligence, 2012, vol. 402, p. 169-181.

[12] E. Adam, D. Trentesaux, et R. Mandiau, « Volatile knowledge to improve the self-adaptation of autonomous shuttles in flexible job shop manufacturing system », Stud. Comput. Intell., vol. 594, p. 219-231, 2015.

[13] T. T. Mezgebe, H. B. El Haouzi, G. Demesure, R. Pannequin, et A. Thomas, «A Negotiation Scenario Using an Agent-Based Modelling Approach to Deal 
with Dynamic Scheduling », in Studies in Computational Intelligence, 2018, vol. 762, p. 381-391.

[14] E. Zimmermann, H. B. El-Haouzi, P. Thomas, R. Pannequin, et M. Noyel, « Using analytic hierarchical process for scheduling problems based on smart lots and their quality prediction capability », in Studies in Computational Intelligence, 2019, vol. 803, p. 337-348.

[15] S. Raileanu, M. Parlea, T. Borangiu, et O. Stocklosa, « A JADE environment for product driven automation of holonic manufacturing», in Studies in Computational Intelligence, 2012, vol. 402, p. 265-277.

[16] O. Cardin et P. Castagna, « Myopia of service oriented manufacturing systems: Benefits of data centralization with a discrete-event observer », in Studies in Computational Intelligence, 2012.

[17] O. Cardin, D. Trentesaux, A. Thomas, P. Castagna, T. Berger, et H. Bril, "Coupling predictive scheduling and reactive control in manufacturing: State of the art and future challenges », Stud. Comput. Intell., vol. 594, p. 29-37, 2015.

[18] M. Gaham, B. Bouzouia, et N. Achour, «An evolutionary simulationoptimization approach to product-driven manufacturing control », in Studies in Computational Intelligence, vol. 544, Springer, 2014, p. 283-294.

[19] M. Li, H. B. El Haouzi, A. Thomas, et A. Guidat, « Fuzzy decision-making method for product holons encountered emergency breakdown in productdriven system: An industrial case », Stud. Comput. Intell., vol. 594, p. 243-256, 2015.

[20] W. Derigent, A. Voisin, A. Thomas, S. Kubler, et J. Robert, « Application of measurement-based AHP to product-driven system control », in Studies in Computational Intelligence, vol. 694, 2017, p. 249-258.

[21] A. Aubry, H. Bril, A. Thomas, et M. Jacomino, « Product driven systems facing unexpected perturbations: How operational research models and approaches can be useful? », in Studies in Computational Intelligence, 2017.

[22] R. F. Babiceanu et R. Seker, «Manufacturing operations, internet of things, and big data: Towards predictive manufacturing systems», Stud. Comput. Intell., 2014.

[23] P. Thomas et A. Thomas, «An approach to data mining for product-driven systems », in Studies in Computational Intelligence, vol. 472, Springer, 2013, p. 181-194.

[24] W. Bouazza, Y. Sallez, N. Aissani, et B. Beldjilali, «A model for manufacturing scheduling optimization through learning intelligent products », Stud. Comput. Intell., vol. 594, p. 233-241, 2015.

[25] E. Zimmermann, H. B. El Haouzi, P. Thomas, R. Pannequin, M. Noyel, et A. Thomas, « A Case Study of Intelligent Manufacturing Control Based on Multiagents System to Deal with Batching and Sequencing on Rework Context », in Studies in Computational Intelligence, 2018.

[26] J. Queiroz, P. Leitão, J. Barbosa, E. Oliveira, et G. Garcia, « An agent-based industrial cyber-physical system deployed in an automobile multi-stage production system », in Studies in Computational Intelligence, 2020, vol. 853, 
p. 379-391.

[27] D. McFarlane, V. Giannikas, A. C. Y. Wong, et M. Harrison, « Intelligent products in the supply chain-10 years on ", in Service orientation in holonic and multi agent manufacturing and robotics, Springer, 2013, p. 103-117.

[28] D. McFarlane, S. Sarma, J. L. Chirn, C. Y. Wong, et K. Ashton, « Auto ID systems and intelligent manufacturing control», Eng. Appl. Artif. Intell., vol. 16, $\mathrm{n}^{\mathrm{0}} 4$, p. 365-376, 2003.

[29] W. Derigent et A. Thomas, "Situation Awareness in Product Lifecycle Information Systems », in Studies in Computational Intelligence, 2018, vol. 762, p. 127-136.

[30] R. Cuthbert, V. Giannikas, D. McFarlane, et R. Srinivasan, « Repair services for domestic appliances », in Studies in Computational Intelligence, 2016, vol. 640, p. 31-39.

[31] W. Derigent et A. Thomas, «End-of-life information sharing for a circular economy: Existing literature and research opportunities », in Studies in Computational Intelligence, 2016, vol. 640, p. 41-50.

[32] K. Främling, S. Parmar, V. Hinkka, J. Tätilä, et D. Rodgers, « Assessment of EPCIS standard for interoperable tracking in the supply chain », in Studies in Computational Intelligence, 2013, vol. 472, p. 119-134.

[33] P. G. Ansola, A. García, et J. de Las Morenas, « IoT visibility software architecture to provide smart workforce allocation", in Studies in Computational Intelligence, 2016, vol. 640, p. 223-231.

[34] S. Kubler, M. Madhikermi, et K. Främling, «QLM messaging standards: introduction and comparison with existing messaging protocols », in Service Orientation in Holonic and Multi-Agent Manufacturing and Robotics, Springer International, 2014, vol. 544, p. 237-256.

[35] Y. Sallez, « The augmentation concept: How to make a product "active" during its life cycle », in Studies in Computational Intelligence, 2012, vol. 402, p. 35-48.

[36] Y. Sallez, «Proposition of an analysis framework to describe the "activeness" of a product during its life cycle part ii: Method and applications », in Studies in Computational Intelligence, vol. 544, Springer, 2014, p. 271-282.

[37] V. Basselot, T. Berger, et Y. Sallez, «Active monitoring of a product: A way to solve the "lack of information" issue in the use phase ", in Studies in Computational Intelligence, vol. 694, 2017, p. 337-346.

[38] F. G. Quintanilla, O. Cardin, et P. Castagna, «Evolution of a flexible manufacturing system: From communicating to autonomous product », in Studies in Computational Intelligence, 2013, vol. 472, p. 167-180.

[39] S. Kubler, W. Derigent, A. Thomas, et É. Rondeau, «Key Factors for Information Dissemination on Communicating Products and Fixed Databases », in Service Orientation in Holonic and Multi-Agent Manufacturing Control, Paris, 2011, vol. 402, p. 89-102.

[40] K. Mekki, W. Derigent, E. Rondeau, et A. Thomas, " Communicating Aircraft Structure for Solving Black-Box Loss on Ocean Crash», in Studies in Computational Intelligence, 2018. 
[41] H. Wan, M. David, et W. Derigent, « A holonic manufacturing approach applied to communicate concrete: Concept and first development », in Studies in Computational Intelligence, 2020.

[42] M. S. Taboun et R. W. Brennan, « Sink node embedded, multi-agent systems based cluster management in industrial wireless sensor networks », in Studies in Computational Intelligence, 2016, vol. 640, p. 329-338.

[43] D. Trentesaux et G. Branger, «Data Management Architectures for the Improvement of the Availability and Maintainability of a Fleet of Complex Transportation Systems: A State-of-the-Art Review », in Studies in Computational Intelligence, 2018, vol. 762, p. 93-110.

[44] D. Trentesaux et G. Branger, «Foundation of the Surfer Data Management Architecture and its application to train transportation », in International Workshop on Service Orientation in Holonic and Multi-Agent Manufacturing, Studies in Computational Intelligence, 2018, vol. 762, p. 111-125.

[45] O. Morariu, C. Morariu, et T. Borangiu, « Resource, service and product: Realtime monitoring solution for service oriented holonic manufacturing systems ", in Studies in Computational Intelligence, 2014, vol. 544, p. 47-62.

[46] N. Tsamis, V. Giannikas, D. McFarlane, W. Lu, et J. Strachan, « Adaptive storage location assignment for warehouses using intelligent products », Stud. Comput. Intell., vol. 594, p. 271-279, 2015.

[47] L. E. Cojocaru, G. Burlacu, D. Popescu, et A. M. Stanescu, « Farm management information system as ontological level in a digital business ecosystem », in Studies in Computational Intelligence, vol. 544, Springer, 2014, p. 295-309.

[48] S. Răileanu, T. Borangiu, et A. Silişteanu, «Centralized HMES with environment adaptation for production of radiopharmaceuticals », in Studies in Computational Intelligence, 2016, vol. 640, p. 3-20.

[49] M. Pătraşcu et M. Drăgoicea, « Integrating agents and services for control and monitoring: Managing emergencies in smart buildings », in Studies in Computational Intelligence, 2014, vol. 544, p. 209-224.

[50] V. Thomson et X. Zhang, "Improving the delivery of a building », in Studies in Computational Intelligence, 2016, vol. 640, p. 21-29.

[51] B. Montreuil, «Toward a Physical Internet: meeting the global logistics sustainability grand challenge », Logist. Res., vol. 3, n 2, p. 71-87, 2011.

[52] E. Ballot, O. Gobet, et B. Montreuil, « Physical internet enabled open hub network design for distributed networked operations », in Studies in Computational Intelligence, 2012, vol. 402, p. 279-292.

[53] A. Rahimi, Y. Sallez, et T. Berger, «Framework for smart containers in the physical internet», in Studies in Computational Intelligence, vol. 640, Springer, 2016, p. 71-79.

[54] N. Krommenacker, P. Charpentier, T. Berger, et Y. Sallez, « On the usage of wireless sensor networks to facilitate composition/decomposition of physical internet containers ", in Studies in Computational Intelligence, vol. 640, Springer, 2016, p. 81-90.

[55] P. Pujo, F. Ounnar, et T. Remous, « Wireless holons network for intralogistics 
service », Stud. Comput. Intell., vol. 594, p. 115-124, 2015.

[56] P. Pujo et F. Ounnar, "Cyber-Physical Logistics System for Physical Internet », in Studies in Computational Intelligence, 2018, vol. 762, p. 303-316.

[57] E. Glaessgen et D. Stargel, « The digital twin paradigm for future NASA and US Air Force vehicles ", in 53rd AIAA/ASME/ASCE/AHS/ASC structures, structural dynamics and materials conference 20th AIAA/ASME/AHS adaptive structures conference 14th AIAA, 2012, p. 1818.

[58] P. Valckenaers, "ARTI reference architecture - PROSA revisited », in Studies in Computational Intelligence, 2019, vol. 803, p. 1-19.

[59] T. Borangiu, E. Oltean, S. Răileanu, F. Anton, S. Anton, et I. Iacob, « Embedded digital twin for ARTI-type control of semi-continuous production processes », in Studies in Computational Intelligence, vol. 853, 2020, p. 113-133.

[60] Q. Lu, X. Xie, J. Heaton, A. K. Parlikad, et J. Schooling, « From BIM towards digital twin: Strategy and future development for smart asset management », in Studies in Computational Intelligence, vol. 853, 2020, p. 392-404.

\section{$6 \quad$ Annexes}

Table 1. List of references extracted from SOHOMA Proceedings, ranked by cluster and year.

\begin{tabular}{|c|c|c|}
\hline Cluster & Title & Year \\
\hline 1 & $\begin{array}{c}\text { A JADE Environment for Product Driven Automation of Holonic Manufactur- } \\
\text { ing }\end{array}$ & 2011 \\
\hline 1 & $\begin{array}{c}\text { Myopia of Service Oriented Manufacturing Systems: Benefits of Data Centrali- } \\
\text { zation with a Discrete-Event Observer }\end{array}$ & 2011 \\
\hline 1 & Service Oriented Architecture for Holonic Isoarchic and Multicriteria Control & 2011 \\
\hline 1 & Viable System Model Approach for Holonic Product Driven Manufacturing \\
Systems. & 2011 \\
\hline 1 & An Approach to Data Mining for Product-driven Systems & 2012 \\
\hline 1 & Product-Driven Control: Concept, Literature Review and Future Trends & 2012 \\
\hline 1 & $\begin{array}{c}\text { An Evolutionary Simulation-Optimization Approach to Product-Driven Manu- } \\
\text { facturing Control }\end{array}$ & 2013 \\
\hline 1 & $\begin{array}{c}\text { A Model for Manufacturing Scheduling Optimization Through Learning Intel- } \\
\text { ligent Products }\end{array}$ & 2014 \\
\hline 1 & $\begin{array}{c}\text { Coupling Predictive Scheduling and Reactive Control in Manufacturing: State } \\
\text { of the Art and Future Challenges }\end{array}$ & 2014 \\
\hline 1 & $\begin{array}{c}\text { Fuzzy Decision-Making Method for Product Holons Encountered Emergency } \\
\text { Breakdownin Product-Driven System: An Industrial Case }\end{array}$ & 2014 \\
\hline 1 & $\begin{array}{c}\text { Volatile Knowledge to Improve the Self-adaptation of Autonomous Shuttles in } \\
\text { Flexible Job Shop Manufacturing System }\end{array}$ & 2014 \\
\hline 1 & Application of Measurement-Based AHP to Product-Driven System Control & 2016 \\
\hline 1 & $\begin{array}{c}\text { Product Driven Systems Facing Unexpected Perturbations: How Operational } \\
\text { Research Models and Approaches Can Be Useful? }\end{array}$ & 2016 \\
\hline
\end{tabular}




\begin{tabular}{|c|c|c|}
\hline Cluster & Title & Year \\
\hline 1 & $\begin{array}{l}\text { A Case Study of Intelligent Manufacturing Control Based on Multi-agents Sys- } \\
\text { tem to Deal with Batching and Sequencing on Rework Context }\end{array}$ & 2017 \\
\hline 1 & $\begin{array}{l}\text { A Negotiation Scenario Using an Agent-Based Modelling Approach to Deal } \\
\text { with Dynamic Scheduling }\end{array}$ & 2017 \\
\hline 1 & $\begin{array}{l}\text { Empowering a Cyber-Physical System for a Modular Conveyor System with } \\
\text { Self-organization }\end{array}$ & 2017 \\
\hline 1 & $\begin{array}{l}\text { Using Analytic Hierarchical Process for Scheduling Problems Based on Smart } \\
\text { Lots and Their Quality Prediction Capability }\end{array}$ & 2018 \\
\hline 1 & $\begin{array}{c}\text { An Agent-Based Industrial Cyber-Physical System Deployed in an Automobile } \\
\text { Multi-stage Production System }\end{array}$ & 2019 \\
\hline 2 & Intelligent Products in the Supply Chain - 10 Years on & 2011 \\
\hline 2 & $\begin{array}{l}\text { Key Factors for Information Dissemination on Communicating Products and } \\
\text { Fixed Databases }\end{array}$ & 2011 \\
\hline 2 & $\begin{array}{c}\text { The Augmentation Concept: How to Make a Product "Active" during Its Life } \\
\text { Cycle }\end{array}$ & 2011 \\
\hline 2 & Assessment of EPCIS Standard for Interoperable Tracking in the Supply Chain & 2012 \\
\hline 2 & $\begin{array}{l}\text { Evolution of a Flexible Manufacturing System: From Communicating to } \mathrm{Au}- \\
\text { tonomous Product }\end{array}$ & 2012 \\
\hline 2 & $\begin{array}{l}\text { Farm Management Information System as Ontological Level in a Digital Busi- } \\
\text { ness Ecosystem }\end{array}$ & 2013 \\
\hline 2 & $\begin{array}{l}\text { Integrating Agents and Services for Control and Monitoring: Managing Emer- } \\
\text { gencies in Smart Buildings }\end{array}$ & 2013 \\
\hline 2 & $\begin{array}{c}\text { Proposition of an Analysis Framework to Describe the "Activeness" of a Prod- } \\
\text { uct during Its Life Cycle (Part I and 2) }\end{array}$ & 2013 \\
\hline 2 & $\begin{array}{l}\text { QLM Messaging Standards: Introduction and Comparison with Existing Mes- } \\
\text { saging Protocols }\end{array}$ & 2013 \\
\hline 2 & $\begin{array}{l}\text { Resource, Service and Product: Real-Time Monitoring Solution for Service } \\
\text { Oriented Holonic Manufacturing Systems }\end{array}$ & 2013 \\
\hline 2 & $\begin{array}{l}\text { Adaptive Storage Location Assignment for Warehouses Using Intelligent Prod- } \\
\text { ucts }\end{array}$ & 2014 \\
\hline 2 & $\begin{array}{l}\text { Manufacturing Operations, Internet of Things, and Big Data: Towards Predic- } \\
\text { tive Manufacturing Systems }\end{array}$ & 2014 \\
\hline 2 & Adaptive storage location assignment for warehouses using intelligent products & 2015 \\
\hline 2 & $\begin{array}{l}\text { Centralized HMES with Environment Adaptation for Production of Radiophar- } \\
\text { maceuticals }\end{array}$ & 2015 \\
\hline 2 & $\begin{array}{l}\text { End-of-Life Information Sharing for a Circular Economy: Existing Literature } \\
\text { and Research Opportunities }\end{array}$ & 2015 \\
\hline 2 & Improving the Delivery of a Building & 2015 \\
\hline 2 & IoT Visibility Software Architecture to Provide Smart Workforce Allocation & 2015 \\
\hline 2 & Repair Services for Domestic Appliances & 2015 \\
\hline 2 & $\begin{array}{c}\text { Sink Node Embedded, Multi-agent Systems Based Cluster Management in In- } \\
\text { dustrial Wireless Sensor Networks }\end{array}$ & 2015 \\
\hline 2 & $\begin{array}{l}\text { Active Monitoring of a Product: A Way to Solve the "Lack of Information" Is- } \\
\text { sue in the Use Phase }\end{array}$ & 2016 \\
\hline 2 & $\begin{array}{c}\text { Communicating Aircraft Structure for Solving Black-Box Loss on Ocean } \\
\text { Crash }\end{array}$ & 2017 \\
\hline 2 & $\begin{array}{l}\text { Data Management Architectures for the Improvement of the Availability and } \\
\text { Maintainability of a Fleet of Complex Transportation Systems: A State-of-the- } \\
\text { Art Review }\end{array}$ & 2017 \\
\hline 2 & $\begin{array}{c}\text { Foundation of the Surfer Data Management Architecture and Its Application to } \\
\text { Train Transportation }\end{array}$ & 2017 \\
\hline
\end{tabular}




\begin{tabular}{|c|c|c|}
\hline Cluster & Title & Year \\
\hline 2 & Situation Awareness in Product Lifecycle Information Systems & 2017 \\
\hline 2 & $\begin{array}{c}\text { A Holonic Manufacturing Approach Applied to Communicate Concrete: Con- } \\
\text { cept and First Development }\end{array}$ & 2019 \\
\hline \hline 3 & On the Activeness of Physical Internet Containers & 2014 \\
\hline 3 & Wireless Holons Network for Intralogistics Service & 2014 \\
\hline 3 & $\begin{array}{c}\text { On the Usage of Wireless Sensor Networks to Facilitate Composition/Decom- } \\
\text { position of Physical Internet Containers }\end{array}$ & 2015 \\
\hline 3 & $\begin{array}{c}\text { the Augmentation Concept: How to Make a Product “Active" during Its Life } \\
\text { Cycle }\end{array}$ & 2015 \\
\hline 3 & Cyber-Physical Logistics System for Physical Internet & 2017 \\
\hline 3 & Integration of Distributed Manufacturing Nodes in Smart Factory & 2018 \\
\hline \hline 6 & ARTI Reference Architecture - PROSA Revisited & 2018 \\
\hline 6 & $\begin{array}{c}\text { Embedded Digital Twin for ARTI-Type Control of Semi-continuous Produc- } \\
\text { tion Processes }\end{array}$ & 2019 \\
\hline 6 & $\begin{array}{c}\text { From BIM Towards Digital Twin: Strategy and Future Development for Smart } \\
\text { Asset Management }\end{array}$ & 2019 \\
\hline
\end{tabular}

